\section{Pollen mit Botschaften}

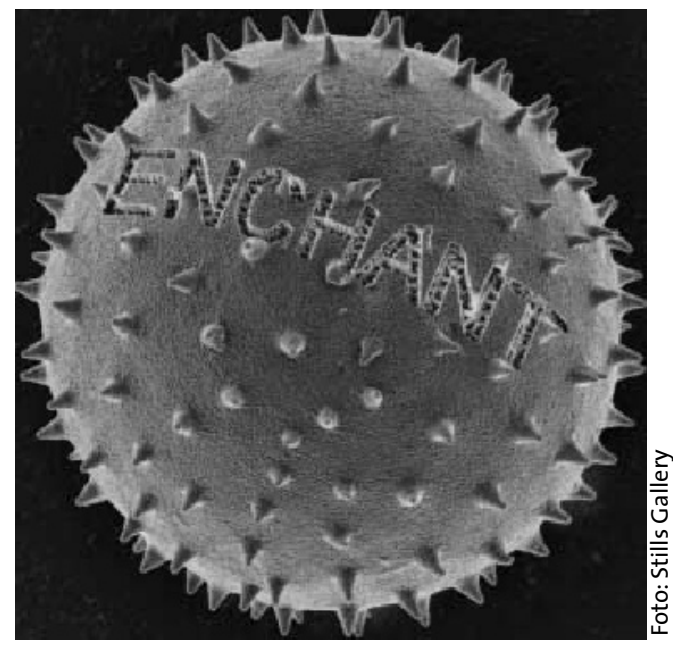

$\mathrm{H}$ ammer und Meißel sind in den Augen von Stephanie Valentin grobe Werkzeuge einer längst vergangenen Epoche. Die australische Künstlerin graviert lieber mit Hilfe von Galliumionen Inschriften in Pflanzenpollen. Ihre mikroskopisch kleinen Kunstwerke, die sie selbst Pollinate nennt, sind in vergrößerter Form und auf Fotopapier belichtet zurzeit in der Stills Gallery in Sydney zu bewundern. Wer sich den Weg ans andere Ende der Welt ersparen will, kann der Galerie auch einen virtuellen Besuch abstatten.

www.stillsgallery.com.au/proofsht/sv

\section{Erdnussallergie nach Hautpflege}

B ritische Pädiater haben neue Risikofaktoren für die Entwicklung einer Erdnussallergie ausgemacht. In einer Kohortenstudie mit 13.971 Vorschulkindern erhöhte die Anwendung erdnussölhaltiger Hautpflegemittel deutlich die Wahrscheinlichkeit, später eine Erdnussallergie zu entwickeln (Odds Ratio 6,8). Ein weiterer unabhängiger Risikofaktor war die Gabe von sojahaltiger Formulaernährung (Odds Ratio 2,6).

Lack G et al. N Engl J Med 2003; 348: 977-85

\section{HEPA hilft nicht}

\section{Nachsitzen für männliche Asthmatiker}

\begin{abstract}
A sthmakranke Männer prägen sich rasch Wissenswertes über ihre Erkrankung ein - doch ebenso schnell vergessen sie es wieder. Bei Asthmatikerinnen verläuft der Wissenszuwachs zwar langsamer, dafür bleibt das Gelernte jedoch länger im Gedächtnis haften. Dies ist das Ergebnis einer Studie der Universität Bremen, bei der 320 Asthmapatienten vor und nach der Schulung sowie 6 und 12 Monate später mit dem „Asthma-Wissen-Test“ befragt wurden. Das signifikante Ergebnis: Während bei den Frauen im
\end{abstract}

Durchschnitt ein flacherer, aber kontinuierlicher Wissensanstieg über den Untersuchungszeitraum zu beobachten war, zeigten Männer einen kurzfristig ausgeprägteren, aber im Zeitverlauf stark abfallenden Schulungseffekt. Inhalte und zeitliche Struktur von Patientenschulungen sollten geschlechtsspezifisch ausgerichtet werden, resümierte Dr. Stephan Mühlig, Bremen. red

44. Kongress der Deutschen Gesellschaft für Pneumologie, München, 26.-29. März 2003

\section{Allergie und Krebs}

A ustralische Epidemiologen dokumentierten 18 Jahre lang die Daten der 3.308 Teilnehmer des Busselton Health Survey, um einem möglichen Zusammenhang zwischen allergischen und malignen Erkrankungen auf die Spur zu kommen. Tatsächlich verdreifachte ein positiver Pricktest gegen Hausstaubmilben im Jahr 1981 das Risiko im Jahr 1999 ein Prostatakarzinom entwickelt zu haben. Neben diesem statistisch signifikanten Ergebnis hatten
Patienten mit allergischen Atemwegserkrankungen in der Anamnese ein reduziertes Risiko, an kolorektalen Tumoren zu erkranken, waren aber dafür häufiger von Leukämie betroffen. $\mathrm{Ob}$ es sich hier um Hinweise auf ungeahnte pathogenetische Zusammenhänge oder doch eher um statistische Zufälle handelt, bleibt fraglich.

es

Talbot-Smith A et al. Am J Epidemiol 2003; 157: 606-12 taubsauger mit verstärkten Staubbeuteln und HEPA-Filtern (highefficiency particulate arrest) sind in experimentellen Untersuchungen in der Lage, Aeroallergene aus der Luft auszufiltern. Leider hat dieses Ergebnis unter Alltagsbedingungen keinen Bestand, wie eine britische Untersuchung zeigt: Während des Saugens mit fünf verschiedenen modernen HEPA-Saugern in einem Katzenhaushalt erhöhte sich die Konzentration von Katzenallergenen in der Luft um den Faktor 3-5 und unterschied sich damit nicht signifikant von der Menge an Allergenen, die ein herkömmliches Gerät aufwirbelte. es

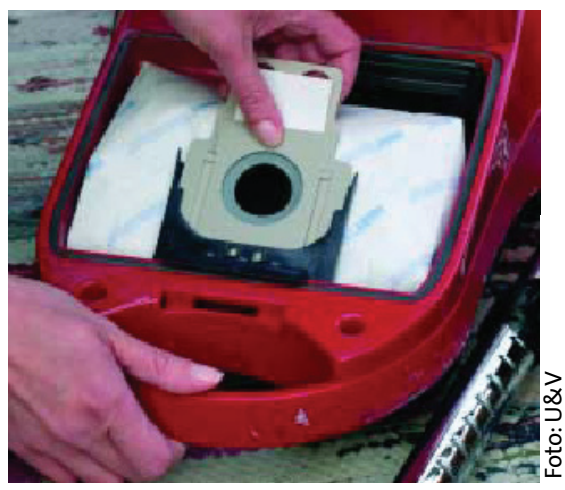

Gore RB et al. J Allergy Clin Immunol 2003; 111: 784-7 